\title{
Changes in lifestyle habits and behaviours are associated with weight loss maintenance in members of a commercial weight loss organisation
}

\author{
R. J. Stubbs ${ }^{1}$, A. McConnon ${ }^{2}$, M. Gibbs ${ }^{3}$, M. Raats ${ }^{2}$ and S. Whybrow ${ }^{3}$ \\ ${ }^{1}$ Slimming World, Alfreton DE55 4UE, ${ }^{2}$ Food, Consumer Behaviour and Health Research Centre, Department of \\ Psychology, University of Surrey, Guildford GU2 7XH and ${ }^{3}$ Food, Consumer Behaviour and Health Research Centre, \\ Faculty of Health and Medical Sciences, University of Surrey, Guildford GU2 7XH
}

This analysis examined the lifestyle correlates of weight loss maintenance in 1428 participants of a slimming organisation, who had been members for a mean \pm SD of $16 \pm 16$ months, had lost $13.8 \% \pm 9.2 \%$ weight and were trying to maintain, or increase, their weight loss during a subsequent 6 month study period.

Data were collected as part of the DiOGenes study ${ }^{(1)}$. Ethical approval was given by the University of Surrey Ethics Committee. Adults were recruited between August 2006 and July 2008 from Slimming World. Subjects completed lifestyle measures at two time points, measurement 1 (M1) at the start of the study and nominally six months later (measurement 2 (M2)). Participants' weights (using calibrated scales) were taken from group records for M1, M2, six months before (measurement 0 ) and when they initially enrolled. They were free to continue following the weight-loss programme as they wished during this study, and there was no intervention other than completing the questionnaires. At M1 and M2 meal frequency (breakfast, lunch and dinner) and snacking between meals were assessed using a 5-point Likert scale (daily to <once/week). Physical activity was assessed using the Baecke Physical Activity Questionnaire ${ }^{(2)}$, which assesses three areas of habitual physical activity level from the previous 12 months, occupational physical activities, physical exercises in leisure (sports), leisure physical activities and a total score. A further question about physical activity level at work was included. Lifestyle habits were assessed using questions adapted from the Self Report of Habit Index ${ }^{(3)}$, which measured the strength of important behaviours relating to weight control, (being physically active, frequent consumption of fruits and vegetables, following a healthy diet and consumption of low fat foods).

Regular lunch consumption was associated with further weight loss $(p=0.002)$. Borderline evidence suggested snacking between meals was associated with weight regain $(p=0.057)$. Dietary habits relating to "eating a healthy diet" or "eating low-fat foods" were related to weight loss when reported at the second measurement, but not at the first and were related to Weight loss maintenance over the six months. The reported strengths of these two habits explained $3.8 \%$ and $0.7 \%$ of the variance in change in percentage body weight, respectively. When looking at change in habit strength over the six months, people for whom "eating a healthy diet", "eating low fat foods" or "eating fruits and vegetables" had become stronger habits tended to have more successful weight changes. The correlations were significant, but very weak $\left(\mathrm{R}^{2}=0.03,0.019\right.$ and 0.01 respectively). People who were more active at the first measurement went on to lose less or gain more weight over the following six months $(p=0.035)$. In contrast, higher "being physically active" habit scores at the second measurement correlated with more weight loss $(p=0.013)$. All significant effects only explained a small proportion of the variance in weight loss maintenance.

These and other data suggest that the habits of successful weight loss maintainers are heterogeneous because people adopt individual lifestyle behaviour profiles. Specific correlates while significant, only explain a small proportion of the variance in weight outcomes for the whole group.

This work was part of the Diet, Obesity and Genes project (www.diogenes-eu.org) funded by the European Commission (contract \#: Food-CT-2005513946) in the Food Quality and Safety Priority of the Sixth Framework Program.

1. Larsen TM, Dalskov S et al. (2010) "The Diet, Obesity and Genes (Diogenes) Dietary Study in eight European countries - a comprehensive design for long-term intervention." Obesity Reviews 11, 76-91.

2. Baecke JAH, Burema, J et al. (1982) "A short questionnaire for the measurement of habitual physical activity in epidemiological studies." American Journal of Clinical Nutrition 36, 936-942.

3. Verplanken B, Orbell S (2003) "Reflections on Past Behavior: A Self-Report Index of Habit Strength." Journal of Applied Social Psychology 33, 1313-1330. 